\title{
El dilema nuclear desde una perspectiva hispano-sueca
}

\author{
Pablo García-García \\ UNIVERSIDAD DE VALLADOLID, VALLADOLID, VALLADOLID, ESPAÑA \\ pabloggarcia@economistas.org
}

\begin{abstract}
Resumen: La energía nuclear es considerada una fuente energética útil y problemática al mismo tiempo. Su utilización ofrece ventajas e inconvenientes que deben ser analizados para comprender el dilema económico, político y social al que se enfrenta una sociedad cuando toma la decisión de configurar su perfil energético. El análisis se plantea desde una perspectiva hispano-sueca, en tanto que España y Suecia son dos ejemplos de las respuestas que una sociedad puede dar ante el mencionado dilema nuclear.
\end{abstract}

Palabras clave: energía nuclear, política energética, España, Suecia

Abstract: Nuclear energy is considered to be a useful and problematic energy source at the same time. Its utilisation offers advantages and inconveniences that must be analysed to understand the economic, political, and social dilemma that a society faces when it takes the decision to set their energy mix. The analysis is set out from a Spanish-Swedish perspective, as Spain and Sweden are two examples of the answers that a society can provide facing the mentioned nuclear dilemma.

Keywords: nuclear energy, energy policy, Spain, Sweden

\section{Introducción}

$C$ l término "energía nuclear" alude al aprovechamiento de la interacción que mantiene unidos los neutrones y los protones del núcleo de un átomo. Sus usos son muy variados: tiene aplicaciones científicas, sanitarias, entre otras. La aplicación más relevante desde la perspectiva de este trabajo es su utilización en la generación de electricidad. Para lograr dicha electricidad, el método más factible y generalizado en la actualidad es la fisión, mediante la cual dichos núcleos se dividen liberando energía calorífica en el proceso de pérdida de masa resultante (CSN, 2015).

Esta forma de obtener energía ha ostentado históricamente una posición relevante en el seno de la Unión Europea. Uno de los Tratados fundacionales, el Tratado Constitutivo de la Comunidad Europea de la Energía Atómica (Euratom), se centró exclusivamente en crear un mercado interno europeo de materiales fisionables, con libertad de movimiento para trabajadores y equipos especializados. Este Tratado de 1958 reconoció en su preámbulo "que la energía nuclear constituye un recurso esen- 
cial para el desarrollo y la renovación de la producción y el progreso de las acciones en favor de la paz" (Euratom, 2012).

La energía nuclear suele ser considerada como una fuente de energía con numerosas ventajas para los consumidores de energía, para la resiliencia del sistema energético y para la sociedad en su conjunto. A pesar de estas ventajas, la energía nuclear es, con certeza, una de las fuentes más polémicas del perfil energético actual. En consecuencia, la planificación energética se halla ante un dilema. Las sociedades deben elegir entre minimizar la importancia de la energía nuclear y buscar sus potenciales ventajas en otras fuentes o formas de explotación-consumo, o bien, estimular la producción nuclear y asumir los potenciales inconvenientes de la misma. Este debate, además de ser relevante desde el punto de vista político-social, es interesante desde el punto de vista académico: es necesario revisar el dilema nuclear en base a hechos probados y a evidencias, y no solo a discursos centrados en la necesidad o la naturalización, con el objetivo de aportar una visión objetiva de la problemática y de los posibles cauces de acción en el futuro (Teräväinen, Lehtonen, \& Martiskainen, 2011).

Por supuesto, las sociedades son heterogéneas entre sí y no existen las mismas preocupaciones, percepciones y prioridades en ellas. Por este motivo, el análisis debe centrarse en estudios de caso específicos. En este trabajo, se han seleccionado dos casos: el caso español, por su cercanía; y el caso sueco, por su comportamiento diferencial en el panorama europeo y en el panorama nórdico, escenarios ambos en los que Suecia puede considerarse un outlier.

Este trabajo va a plantear el dilema nuclear atendiendo a las ventajas e inconvenientes potenciales y reales de la energía nuclear, con el fin de mostrar la disyuntiva a la que se enfrenta la política energética. Con el fin de ilustrar este debate teórico de una manera más cercana y aplicada, se recurrirá a los dos casos de estudio mencionados, España y Suecia. Finalmente, se sintetizarán las principales conclusiones alcanzadas y se tratarán de detectar oportunidades futuras de investigación.

\section{La implantación de la energía nuclear}

La revisión del proceso de introducción de la energía nuclear como una fuente adicional del perfil energético es vital para comprender su situación actual.

En España, fue un proceso complejo (CSN, 2015; Espejo Marín, 2002). Se inició a finales de 1948 mediante una colaboración entre científicos y militares en el seno del Laboratorio de Investigación del Estado Mayor de la Armada. El conocimiento necesario para enfocar el Laboratorio hacia las aplicaciones nucleares procedió de una donación de materiales científicos efectuada por la Embajada en España de los Estados Unidos de América. Esta colaboración dio lugar a la creación de la Junta de Investigaciones Atómicas, encargada de formar al equipo humano necesario para hacerse 
cargo de los procesos nucleares, analizar los potenciales yacimientos de materiales fisionables y protocolizar la gestión de todas las fases del proceso de producción. En los primeros momentos tras su creación, la Junta tuvo que recurrir a instituciones extranjeras para captar el saber hacer requerido por tales actividades, entonces inusitadas en territorio español. La Junta de Investigaciones pasó posteriormente a ser conocida como la Junta de Energía Nuclear, en conjunción con la Sociedad de Estudios y Proyectos de Aleaciones Especiales. En la actualidad, la Junta está dividida en dos organismos: el Consejo de Seguridad Nuclear y el Centro de Investigaciones Energéticas, Medioambientales y Tecnológicas, nombres que evidencian la trascendencia de la tipología energética objeto de estudio.

A medida que el proyecto de implantación fue evolucionando, las instituciones mencionadas, originariamente especializadas en la energía nuclear, fueron ampliando su campo de acción a otras áreas de las ciencias, la minería y la metalurgia. Estos esfuerzos iniciales no dieron lugar a un proyecto de aplicabilidad concreta hasta finales de la década de 1960, periodo en el que se puso en funcionamiento el primer reactor español, llamado JEN-I (Madrid), junto con la Planta Caliente M-1, la instalación encargada del tratamiento de los productos y subproductos nucleares. A principios de esta década, también se creó la primera Ley sobre la Energía Nuclear.

Tras los resultados del JEN-I, coincidiendo con el inicio de la década de 1970, se instaló y activó la primera generación de centrales nucleares propiamente dicha, constituida por las instalaciones de Almonacid de Zorita/José Cabrera, Vandellós I y Santa María de Garoña. Paralelamente, se creó una Empresa Nacional de Uranio, cuyo cometido era gestionar los aprovisionamientos del mineral para cubrir las necesidades de las centrales.

En la década de 1980 se activaron las instalaciones de Almaraz, Ascó, Cofrentes, Vandellós II y Trillo, al tiempo que se inauguró la Empresa Nacional de Residuos Radioactivos, con el cometido de gestionar los subproductos radiactivos del proceso de obtención de energía. El principal punto de gestión se estableció en la década de 1990 en el Centro de Almacenamiento de Residuos de Baja y Media Actividad de El Cabril.

Tanto la primera generación de centrales nucleares como la segunda se situaron en el centro y norte peninsular. Esta disposición geográfica fue parte del plan de minimización de riesgos y de optimización de actividades de producción: la región geográfica citada tiene menor actividad sísmica que el resto de la península, lo que reduce los peligros de daño a las instalaciones, y además, posee los cauces de los ríos Ebro y Tajo para abastecer la refrigeración interna de los reactores (Espejo Marín, 2002).

El establecimiento de centrales nucleares no fue el único paso relevante en la implantación de la fuente nuclear: se precisó un combustible. El combustible más extendido es el uranio tratado, debido a su naturaleza fisionable y altamente calo- 
rífica. Los primeros experimentos nucleares en suelo español y la primera generación de centrales se sirvieron de recursos nacionales para abastecer sus necesidades de uranio. Estos recursos procedieron fundamentalmente de las minas de uranio de Saelices el Chico hasta el año 2001, momento en el que empezó a importarse a causa del agotamiento de la mina salmantina y al diferencial de rentabilidad que favorece al uranio extranjero. Si bien dicha mina no abastecía por completo las necesidades de las centrales, de hecho se estima que aproximadamente cubría el 30\% de las mismas (Espejo Marín, 2002) y existían otros yacimientos en Extremadura y Cataluña (Martínez-Alegría, Sanz, Oliveira, Montequi, \& Campos, 2014), la restricción de su explotación y el consiguiente incremento de la dependencia física exterior incrementó la vulnerabilidad energética española.

Además de la infraestructura y el combustible, el tercer elemento determinante es la política. Las primeras centrales se construyeron siguiendo el Plan Eléctrico Nacional de 1972, que pronto quedó relegado a un plano secundario con la crisis petrolera de la década de 1970. La crisis exigió la elaboración de un Plan Energético Nacional en 1975, cuya prioridad era reducir la dependencia de esta fuente fósil. El instrumento considerado más apropiado para tal misión fue la energía nuclear, que debería fomentarse hasta alcanzar el 56\% de la producción eléctrica nacional. A pesar de que el Plan se ideó con una validez de diez años, fue sustituido por un nuevo Plan en 1978 que rebajó las expectativas electronucleares hasta un objetivo del 37\%. De nuevo este Plan no cumplió la década y en 1984 fue sustituido por otra estrategia energética que tenía como meta limitar la relevancia nuclear, objetivo que quedó confirmado en el siguiente Plan de 1992 y en la Ley de Ordenación del Sistema Eléctrico Nacional de 1994. Esto se tradujo en paralizaciones de obras para aumentar la capacidad nuclear, bien definitivas, o bien en moratoria. La Ley del Sector Eléctrico de 1997 convirtió la moratoria de 1994 en una paralización definitiva, que comportó la creación de unos pagos compensatorios a las empresas energéticas responsables de las centrales descartadas (Espejo Marín, 2002; Mir, 1999). Actualmente, estas empresas son principalmente Iberdrola, Endesa, Gas Natural Fenosa y EDP (WNA, 2018). La moratoria supuso un freno a los ambiciosos objetivos de la década de 1970 (Rubio-Varas \& De la Torre, 2017). En unos años, los planes políticos pasaron de fomentar el avance de la energía nuclear en base a sus ventajas potenciales a paralizar, e incluso desmantelar, los progresos logrados. Esta doble vertiente revela la existencia del mencionado dilema nuclear que suele ir ligado a las presiones sociales e industriales y a las opiniones del Gobierno. En esta línea, cabe destacar que el país ha introducido un impuesto sobre la energía nuclear.

En Suecia, la energía nuclear también se introdujo como resultado de los proyectos del Ejército, con los primeros experimentos ejecutados hacia la década de 1950 
(Larsson, 1985). La empresa pública encargada de gestionar el proceso fue Atomenergi $A B$ (WNA, 2018). La principal razón para fomentar la búsqueda de fuentes alternativas en aquel momento fue el encarecimiento de la energía hidroeléctrica, producida en los ríos del norte. Después de medio siglo (1900-1950) de explotación de los ríos septentrionales, su preservación con fines energéticos se había encarecido demasiado como para continuar utilizándolos. Al mismo tiempo, la principal compañía eléctrica del país impuso a los sistemas locales de producción de energía un precio elevado para asegurar el abastecimiento en caso de deterioro del suministro (Kåberger, 2007).

Entre 1954 y 1960 se establecieron tres reactores en pruebas en Estocolmo y en Nyköping. La primera central fue la de Ågesta, creada para abastecer de calor y electricidad a la capital. Se inauguró en 1964, con tres años de retardo y un sobrecoste del $400 \%$ sobre lo presupuestado. Fue clausurada diez años después por su inviabilidad financiera (Leijonhufvud, 1994). En 1970 hubo un intento de construir la central de Marviken, que nunca llegó a funcionar. En ese periodo, se puso en marcha Oskarshamn, con dos reactores, y Barsebäck con otros dos reactores. En 1975 y 1976 se inauguraron los dos primeros reactores de la central de Ringhals ( $W N A, 2018$ ). La juventud de la tecnología, unida a una sobreestimación de sus efectos sociales fruto del deseo de reducir el precio de la electricidad y de las presiones de la oposición, propició un desajuste de los costes y una desviación de la demanda estimada respecto a la demanda real. El coste resultante era tan elevado en comparación con las alternativas para generar electricidad que su mantenimiento se convirtió en un problema político. El problema político fue trasladado a la ciudadanía mediante la celebración de un referéndum en marzo de 1980. La consulta se planteó en términos confusos pero garantistas: todas las opciones limitaban cuantitativamente la utilización de la fuente o la rechazaban por completo. La opción más votada proponía contar con un máximo de 12 reactores con duración no superior a 25 años (Kåberger, 2007). Con el respaldo de las urnas, en 1980 se inauguró Forsmark; en 1981, el segundo reactor de Forsmark y el tercero de Ringhals; en 1983, el cuarto de Ringhals; y en 1985, el tercero de Oskarshamn. Forsmark, Ringhals y Oskarshamn son las centrales activas actualmente (WNA, 2018). Después de la proliferación de reactores descrita, se comprobó que las previsiones públicas sobre la necesidad de promocionar la energía nuclear fueron erróneas. La realidad se aproximaba más a las previsiones proporcionadas por los grupos ecologistas (Tengström, 1990).

En la década de 1990, coincidiendo con el cumplimiento del plazo acordado de utilización de los reactores, la Ministra competente ordenó el cierre de los reactores más antiguos. Este movimiento desencadenó una oposición por parte de la industria sueca, altamente intensiva en consumo e interesada en el ahorro de costes que gene- 
rarían los retardos del cierre nuclear. De hecho, el sector industrial financió la constitución de grupos de presión políticos. El coste de las campañas de presión era varias veces inferior al coste que debería afrontar la industria si los reactores eran progresivamente desconectados. Tenían poco que perder y mucho que ganar. Sus tareas de presión política dieron resultado y el cierre previsto no se produjo. Esta vulneración del "contrato social" nuclear es el germen de la prevalencia de la fuente energética en el perfil sueco. La prórroga quedó refrendada por el Parlamento a finales de la década, aunque se decretó el cierre de los reactores más antiguos de Barsebäck, lo que originó una compensación pública a la empresa responsable, y se supeditó el cierre de otros reactores al avance en la introducción de renovables y a la mejora de la eficiencia. Este resultado fue recibido con satisfacción por los grupos de presión, que consiguieron sus objetivos y, además, pagos compensatorios por el cierre de las instalaciones envejecidas. Después de esta decisión, algún miembro del Ejecutivo fue contratado por la industria energética (Kåberger, 2007), al igual que ocurrió en España. La propietaria mayoritaria actual de las centrales es la empresa Vattenfall (WNA, 2018).

En la primera década del siglo XXI, se produjo la llegada al Gobierno de una coalición de partidos con una postura más bien favorable a la energía nuclear. En coherencia, su decisión fue mantener el statu quo de la fuente en el perfil nacional. Su principal argumento era la positiva adecuación de la tecnología nuclear para satisfacer los objetivos medioambientales (Alfsen \& Eskeland, 2007). El mandato de la coalición se prolongó durante dos legislaturas hasta la presente década, en la que fue sustituida al frente del Gobierno por otra coalición de partidos con una postura más reticente a la energía nuclear (Holmberg \& Hedberg, 2011). En la actualidad, el Gobierno desearía sustituir la fuente nuclear por energías renovables, pero reconoce la necesidad de mantenerla en su perfil. Su desmantelamiento debería ser progresivo para que todos los agentes puedan adaptarse a los cambios. El país mantiene un impuesto sobre la energía electronuclear (WNA, 2018).

Respecto a la utilización de materiales fisionables, Suecia ha internacionalizado todo el proceso de extracción y procesamiento de uranio (Skala, 2008). Suecia planteó sus proyectos nucleares iniciales pensando en el autoabastecimiento, que fue descartado en la década de 1960, cuando los costes del proceso de obtención del uranio nacional hacían inviable su extracción y procesamiento (Fjæstad, 2013). El incremento de costes internos hizo más atractiva la idea de importarlo. Desde entonces, se ha importado el uranio desde Estados Unidos, principalmente, mediante un acuerdo formal. Asimismo, se ha establecido un acuerdo con otras empresas europeas para el procesamiento del uranio natural. A comienzos del nuevo siglo, las dinámicas de precios de los mercados internacionales de uranio motivaron la propuesta de retornar a una Suecia autosuficiente y se iniciaron estudios sobre posibles yacimientos. El 
principal hallazgo fue el potencial de Häggån, el segundo mayor yacimiento de uranio del mundo por explotar, cuyo estudio de viabilidad promete reducciones elevadas de costes por sustitución de importaciones (Aura Energy, 2012).

Como miembros de la Unión Europea, tanto España como Suecia han tenido que cumplir a lo largo del proceso las normativas comunes en materia de energía nuclear (Consejo de la Unión Europea, 2011, 2014b, 2014a).

\section{Las ventajas de la energía nuclear}

En ambos casos objeto de estudio, la ventaja más citada hasta el momento para justificar los beneficios de la fuente nuclear es su capacidad para incrementar la seguridad energética. El concepto de seguridad energética implica la disponibilidad, accesibilidad, asequibilidad y aceptabilidad del suministro (APERC, 2007), la baja probabilidad de daños sobre los valores adquiridos (Baldwin, 1997) o la baja vulnerabilidad de los sistemas energéticos vitales (Cherp \& Jewell, 2014). Así, la utilización de la energía nuclear en el mix energético está asociada al deseo de alcanzar el autoabastecimiento y de poseer un suministro garantizado (Segoviano Monterrubio, 2011) y más asequible. En el caso español, se ha observado que los esfuerzos potenciadores de la energía nuclear coincidieron con las crisis del petróleo, en un intento por reducir la cuota de uso del combustible fósil y la dependencia externa. Un incremento de la energía nuclear diversifica el perfil energético e implica la producción interna y la sustitución de flujos energéticos a través de corredores internacionales peligrosos. En el caso sueco, coincidieron con el deterioro en la rentabilidad de la fuente hidroeléctrica y con el encarecimiento de la producción a nivel local.

Paralelamente, en un contexto de creciente demanda de energía a nivel mundial, la energía nuclear es considerada una fuente viable y preferencial debido a su gran capacidad de producción y a la moderada cantidad de combustible requerido para su funcionamiento (Grandin, Jagers, \& Kullander, 2010). Se ha demostrado la existencia de una relación positiva, bidireccional a corto plazo y unidireccional a largo plazo, entre el crecimiento de la economía y la utilización de energía nuclear, tanto en el caso español como en el sueco (Apergis \& Payne, 2010). La mencionada eficiencia hace que su precio sea bajo en relación con otras fuentes energéticas, así como menos volátil y más competitivo. En lo concerniente a los precios, la energía nuclear sirve para compensar los imprevistos causados por otras fuentes, como los combustibles fósiles importados, cuyos precios quedan a merced de factores a priori no controlables desde una perspectiva nacional y que lastran la rentabilidad de la producción. Empíricamente, se ha demostrado que existe una correlación positiva entre los precios del petróleo y la producción nuclear, lo que confirma su potencial de sustitución (Lee \& Chiu, 2011). 
Es, asimismo, una fuente energética que dota de flexibilidad al sistema y previene su vulnerabilidad. La flexibilidad se deriva de su capacidad para variar su producción en el corto plazo con menos condicionantes que otras fuentes. Su fiabilidad se traduce en la escasa probabilidad de sufrir cortes de suministro o de soportar deterioros de calidad en el abastecimiento, como periodos de intermitencia (Jenkins et al., 2018). Por estos motivos, la electronuclear es un complemento perfecto para sistemas energéticos que potencian el uso de renovables, como el sueco, altamente vulnerables ante condicionantes como los cambios meteorológicos (Suman, 2018).

Respecto a la sostenibilidad, la energía nuclear no sólo es presentada como un complemento de las fuentes renovables, sino como una fuente compatible con los objetivos de transición hacia una economía neutra en carbono por su escaso nivel de emisiones (NEA, IEA, \& OECD, 2015). De hecho, se ha propuesto su utilización como un instrumento para combatir el cambio climático (Fawcett et al., 2015).

Finalmente, se trata de una fuente segura no sólo en el sentido energético, sino también en el sentido operativo. La probabilidad de sufrir un accidente nuclear, aunque no es nula, está minimizada por una serie de barreras tecnológicas que dificultan el escape de radiación al exterior, previniendo daños sobre la salud o el medioambiente (Högberg, 2013). Estas instalaciones seguras también son compactas y duraderas. Ocupan un espacio pequeño en relación a la cantidad de energía que producen en unas instalaciones cuya vida útil se prolonga de manera continuada en base al desarrollo tecnológico. Actualmente, los reactores tienen una vida útil de 60 años, que podría ser prorrogable incluso hasta los 80 años (Allen, Busby, Meyer, \& Petti, 2010). Su eficiencia y durabilidad compensan los costes de producción.

\section{Los inconvenientes de la energía nuclear}

En contraposición a las ventajas de la energía nuclear, se van a exponer los inconvenientes de la fuente energética en cuestión. A este respecto, no van a surgir nuevas peculiaridades distintas de las ya revisadas, sino que los inconvenientes pueden obtenerse por simetría: todas las ventajas recogidas presentan alguna problemática asociada.

Si bien la energía nuclear, al tratarse de una fuente de producción eminentemente nacional, reduce los riesgos geopolíticos en el transporte de flujos energéticos, la seguridad de abastecimiento puede ponerse en duda si el combustible nuclear es importado. Si el combustible procede del exterior, el riesgo energético deviene de nuevo considerable. Se ha comprobado que la energía nuclear se emplea para reducir la dependencia exterior y la volatilidad de los precios. Un problema de importación puede desvirtuar estas ventajas, aunque existen analistas que consideran este riesgo 
limitado por la disparidad de países que concentran las reservas de combustible (Bertel, 2005).

Otro inconveniente es el precio comparativo. Si bien la energía nuclear es de las fuentes energéticas más baratas, no es la más barata de todas las existentes. La energía renovable es más barata, aunque tiene el peligro de intermitencia del suministro.

Se ha mostrado que las instalaciones nucleares son seguras en el sentido operativo del término. Sin embargo, el riesgo no es inexistente. Un accidente nuclear puede poner en riesgo el suministro de energía, lo que podría evitarse mediante la redundancia de instalaciones y la diversificación (Kosai \& Unesaki, 2017), aunque encarecería la generación de energía. Más gravemente que el abastecimiento, podría resultar afectada la salud de los seres vivos en amplios rangos de distancias respecto al lugar del accidente y en varias generaciones (Lyman, 2011). Los dos desastres nucleares de mayor impacto han sido los ocurridos en Chernóbil (URSS-Ucrania) y Fukushima (Japón), con significativa influencia sobre el panorama energético europeo (Wittneben, 2012). Ambos se han saldado con mortandad directa e indirecta, proliferación de enfermedades con impacto intergeneracional, riesgos para la seguridad de los alimentos, terrenos contaminados durante décadas y movimientos de población (Steinhauser, Brandl, \& Johnson, 2014). Los daños de los accidentes nucleares tienen un coste económico inestimable.

Las perspectivas optimistas acerca de la durabilidad de las instalaciones también se pueden poner en duda (San Martín, Muñoz, \& Lorca, 2014). Los materiales están sometidos a un considerable estrés fruto de las altas temperaturas y del ambiente corrosivo en el interior del reactor, cuyo deterioro debe ser evitado mediante innovaciones tecnológicas (Allen et al., 2010). Una vez que la instalación abandona sus operaciones, surge el problema de sustituir la antigua producción por otras fuentes, que suelen ser más contaminantes, con el impacto medioambiental que esto conlleva (Davis \& Hausman, 2016).

La salud de los ecosistemas no sólo peligra ante accidentes puntuales, sino también ante el almacenamiento continuado de los residuos nucleares, dando lugar a problemas medioambientales y de equidad intergeneracional. Estrechamente vinculado al medioambiente, existen indicios que ponen en duda la capacidad de la energía nuclear como complemento de las políticas de sostenibilidad. La energía nuclear, si bien favorece a priori los objetivos medioambientales, está lejos de mostrar un desarrollo que pueda considerarse sostenible (Pearce, 2012)Su sostenibilidad depende de la innovación tecnológica aplicada al proceso de obtención de energía, a la mitigación de riesgos y a la gestión de los subproductos radiactivos. En esta línea, existen barreras al desarrollo de la energía nuclear vía tributación (Hejazi, 2017). 
Usualmente, el debate nuclear suele focalizarse en el proceso de producción de energía y en la gestión de los desechos, relegando la obtención de combustibles nucleares a un segundo plano, a pesar de ser relevante. Debe prestarse atención al combustible por excelencia: el uranio. Este elemento presenta problemas en su obtención, en su procesamiento y en sus perspectivas de futuro. La obtención del uranio ha estado históricamente marcada por condiciones laborales precarias, afanes colonialistas, su utilización para fines no pacíficos y tensiones geopolíticas (Zoellner, 2009). En la propia obtención, e incluso posteriormente, se produce una liberación de radiación al ambiente, causando la contaminación del lugar de extracción y de localizaciones adyacentes. En España, la disponibilidad de uranio más relevante se localiza en el noroeste, coincidiendo con los principales lugares de abastecimiento mencionados. En estos lugares, se ha detectado una radiación superior a la esperada que puede resultar nociva (Carvalho et al., 2007; Santos-Francés et al., 2018). Respecto a su procesamiento, es destacable su emisión de gases contaminantes. El bajo nivel de emisiones que registran las operaciones de producción de energía no debe enmascarar las emisiones que se producen en los procesos de tratamiento del combustible, un paso imprescindible para su aprovechamiento energético posterior (Mudd \& Diesendorf, 2008), así como en el procesamiento de los residuos nucleares (Lenzen, 2008). En cuanto a su futuro, hay que perfilar el posible impacto de un agotamiento del uranio o de una pérdida de rentabilidad.

La opinión pública es, asimismo, reticente a asimilar un desarrollo nuclear. Se ha encontrado que estas reticencias están eminentemente provocadas por el riesgo percibido de accidente nuclear. Este riesgo percibido suele estar sobrevalorado respecto a la situación de riesgo objetivo, lo que genera una utilidad negativa para los consumidores de energía y un coste social que puede afectar a las condiciones económicas de las plantas nucleares en el futuro (Huhtala \& Remes, 2017). Estas reticencias están significativamente correlacionadas con factores demográficos y geográficos (BaltaOzkan \& Le Gallo, 2018). Los motivos oficiales de los principales movimientos antinucleares para rechazar esta energía no sólo se centran en el riesgo de accidente, sino también en la contaminación radiactiva, en la generación de subproductos difícilmente procesables y en lo cara que resulta en términos monetarios si se compara con las renovables (Teule, 2011). Sea cual sea la naturaleza de la reticencia, tal postura es una restricción para la fuente de energía a tener en cuenta.

\section{Indicadores de evolución y situación}

Después de plantear el proceso de introducción de la fuente energética y los componentes que dan lugar al dilema nuclear, es momento de caracterizar la evolución de 
la misma y su situación actual de forma cuantitativa. Los indicadores seleccionados están alineados con las ventajas y desventajas encontradas.

\subsection{La energía nuclear en el perfil energético}

Con el fin de contextualizar la situación de la energía nuclear, se va a comenzar determinando su participación en el perfil energético (IEA, 2017).

A nivel mundial, la nuclear está entre las fuentes menos utilizadas (4,9\%). Sin embargo, su uso es mayor que el de las energías renovables. La presencia de la energía nuclear ha aumentado desde la década de 1970 (0,9\%). En las últimas dos décadas su crecimiento parece haberse estancado. Entre los países con mayor nivel de desarrollo económico, su participación aumenta hasta 9,8\% en 2016. Entre los mayores productores mundiales destaca España (2,2\%), en noveno puesto. España asciende al puesto quinto $(20,6 \%)$ si se observa la prevalencia nuclear en la producción eléctrica nacional. Sin embargo, no destaca por su capacidad instalada neta, donde su presencia en el ranking es sustituida por Suecia. En la Unión Europea (EUROSTAT, 2018), las posiciones son mixtas: existen catorce países que generan energía nuclear y catorce países que no lo hacen. El líder europeo es Francia, con amplios diferenciales de producción en las clasificaciones comunitarias y frecuentemente mencionado como ejemplo en España, por su cercanía. No obstante, la aparición de Suecia entre los mayores usuarios comunitarios, al mismo nivel que España (7\%) a pesar de sus disparidades socioeconómicas, alienta la comparativa.

En España (EUROSTAT, 2018), se observa una tendencia lateral desde 1990 (cuando se tienen datos comparables). La tendencia lateral conjuga tres tendencias inferiores: creciente (1990-2004), altamente decreciente (2004-2009) y moderadamente creciente (2013-2016). El crecimiento nuclear se observa en la década de 1990, especialmente a partir de 1998, y se prolonga hasta 2004. A partir de 2004 se fue reduciendo la producción nuclear paulatinamente, fluctuando en años alternos, pero con una marcada tendencia negativa hasta 2009, momento en el que se constata el impacto de la crisis sobre el consumo energético y la producción nuclear. La tendencia iniciada en 2004 más el impacto coyuntural de la crisis dejó la producción en niveles situados por debajo de los observados en la década de 1990. Después de 2009 se recuperó el patrón de evolución alterno, y se retomaron los niveles de producción anteriores a la crisis más un ligero incremento. A partir de 2011 se cortó el patrón de evolución y se ha registrado un crecimiento leve pero sostenido de la fuente nuclear, con un repunte constatable a partir de 2013. En 2016, la producción nuclear está en una posición intermedia entre el máximo de 1998-2004, aproximadamente 16000 TOE, y el mínimo de los años 90 y de la crisis de 2009, aproximadamente 14000 TOE. 
En Suecia (EUROSTAT, 2018), se observa una tendencia decreciente desde 1990. La tendencia decreciente abarca un periodo de evolución lateral hasta el año 2000, que pasa a registrar un notable decrecimiento. Acto seguido, se recuperan los niveles y se retoma la tendencia lateral hasta 2004 , cuando se inicia una marcada tendencia decreciente hasta la crisis. En la recuperación, se observa un retorno a los niveles de producción previos a 2009 con una caída a partir de 2013, que tiende a solucionarse en 2015. En 2016, la producción nuclear, en torno a 16000 TOE, se encuentra entre los niveles mínimos de la serie consultada, que oscila entre los aproximadamente 20000 TOE de 2004 y los 13500 TOE de 2009.

\subsection{Origen y precio de los materiales fisionables}

La fuente nuclear presenta una dependencia exterior elevada. España utilizó los yacimientos de uranio del oeste del país hasta que paralizó su explotación y comenzó a importarlo del exterior. Suecia también intentó autoabastecerse, pero acabó recurriendo igualmente a la importación.

Respecto al material más empleado, el uranio, las necesidades deben ser cubiertas mediante importaciones procedentes de países externos a la Unión Europea. Los principales proveedores de España son Nigeria, Canadá, Rusia, Kazajistán, Australia, Namibia, Estados Unidos, y Uzbekistán (Agencia de Suministro Euratom, 2016). Otros proveedores son Francia, Groenlandia, Noruega, India, Egipto, Sudáfrica y Venezuela (SNE, 2018). Históricamente, el principal proveedor de Suecia ha sido Estados Unidos (Fjæstad, 2013). Los proveedores africanos, asiáticos y Venezuela presentan una elevada conflictividad geopolítica (Institute for Economics and Peace, 2017), que afecta menos a Suecia que a España por recurrir a Estados Unidos.

Tomando como referencia el octaóxido de triuranio, la tipología más empleada, se puede observar un incremento continuado de los precios en la última década (Observatorio Nuclear Europeo, 2018). En el año 2016, el precio por kilogramo era de 86,62 EUR en contratos multianuales y de 88,56 EUR en spot. El máximo de la década se alcanzó en 2015 con 94,30 EUR y 88,73 EUR, respectivamente, lo que contrasta con las cotizaciones de principios de la década de los 2000, que no sobrepasaron los 39 EUR en contrato ni los 26 EUR en spot.

Otros materiales que podrían estudiarse son el cobalto, el torio y el plutonio, y otro procedimiento que podría analizarse es el de fusión nuclear, pero por motivos de uso y difusión se le ha prestado atención al uranio fisionable. 


\subsection{Duración de las instalaciones}

En el proceso de producción, es necesario analizar estadísticamente la infraestructura. La duración se va a medir por el número de años operativos conocidos hasta el momento presente de los reactores y las centrales activos.

En la actualidad, existen 6 centrales nucleares y 8 reactores en territorio español (Ministerio de Energía Turismo y Agenda Digital de España, 2018). El tiempo medio de servicio de un reactor nuclear español es de 35,25 años y el de una central, de 35,66 años. Destaca Trillo con 30 años y Garoña con 47. Todas las centrales fueron inauguradas en la década de 1980 a excepción de Santa María de Garoña (1971), la más antigua del país. La más reciente es Trillo (1988). De acuerdo a sus licencias actualizadas, Almaraz y Vandellós II tienen permiso para operar hasta 2020; Ascó y Cofrentes, hasta 2021; y Trillo, hasta 2024 (WNA, 2018). El Gobierno formado en 2018 modificará estos plazos para clausurar las centrales cuando cumplan 40 años ( in confirmación oficial, 2018). Todas las centrales cuentan con reactores de agua a presión excepto Cofrentes, con un reactor de agua en ebullición. Cada uno de los reactores genera aproximadamente el $13 \%$ de la producción nuclear total, a excepción de la más antigua, Garoña, que produce ligeramente menos del 6\%.

En Suecia, existen 3 centrales nucleares y 8 reactores (WNA, 2018). El tiempo de funcionamiento medio de un reactor es de 37,25 años y de una central, 38 años. Destaca Ringhals con 43 años y Oskarshamn con 33. Todas las centrales fueron inauguradas en la década de 1980 a excepción de los dos primeros reactores de Ringhals $(1975,1976)$. Estos reactores más antiguos tienen permiso para funcionar hasta 2019 y 2020; los tres reactores de Forsmark, hasta 2040, 2041 y 2045; los dos más recientes de Ringhals hasta 2041 y 2043; y Oskarshamn, hasta 2035 como mínimo. Estos datos indican que la intención de los planificadores suecos es considerar una vida útil de 44 años para los reactores antiguos y de exactamente 60 años para los reactores nuevos. De los 8 reactores, 5 son de agua en ebullición y 3, de agua a presión. La central más antigua, Ringhals, genera el $44 \%$ de la producción nuclear del país, seguida por Forsmark, con el 39\% y Oskarshamn con el 17\%.

\subsection{Emisiones}

En el año 2015 se constataron emisiones asociadas a la producción de energía nuclear en centrales españolas de 853,821 kt de dióxido de carbono (Muñoz Mansilla, 2015). El 27,6\% de las mismas se produjeron en Almaraz, el 25,4\% en Ascó, el 16,5\% en Cofrentes, el 16\% en Vandellós y el 14,5\% en Trillo. Comparando producción y emisiones, se puede determinar que apenas existen diferencias entre la eficiencia de las centrales y de los reactores españoles. Se estima que la producción de energía nuclear 
en España evita la emisión de entre 45 y 55 millones de toneladas de dióxido de carbono al año procedentes de otras fuentes energéticas más contaminantes (de las Heras Abás, 2016; Foro de la Industria Nuclear Española, 2016).

En el caso de Suecia, la producción de energía nuclear evita emisiones de dióxido de carbono de aproximadamente 54 millones de toneladas, tomando como referencia la vida media calculada anteriormente de 37,25 años por reactor y comparando las emisiones con un escenario de utilización masiva de fuentes altamente contaminantes (Qvist \& Brook, 2015). Utilizando los factores de Qvist y Brook (2015) se ha estimado una emisión de dióxido de carbono anual asociado a la producción de energía nuclear de aproximadamente $280 \mathrm{kt}$ en 2015. Si se utilizan factores menos optimistas, las emisiones ascienden a los cuatro dígitos (WWF, 2009).

Es necesario tener presente que los datos aportados se refieren al proceso de producción de energía nuclear y a la comparativa de emisiones con fuentes contaminantes sustitutivas. El procesamiento del combustible también comporta emisiones de efecto invernadero que no son recogidas en estos datos. Las ventajas comparativas enunciadas desaparecen si el contraste se lleva a cabo sobre las energía renovables en vez de sobre las fuentes más contaminantes.

\subsection{Residuos}

La gestión de residuos más común en España es la de media y baja intensidad radiactiva. La modalidad principal es la de almacenamiento en superficie (Ministerio de Energía Turismo y Agenda Digital de España, 2018). En el año 2016 se procesaron $1099 \mathrm{~m}^{3}$, el 55\% de los cuales de baja y media intensidad y el 45\% restante de muy baja intensidad. Estrictamente ligados a la energía nuclear, es decir, descontando los residuos generados por otros usos, como los sanitarios o los industriales, se procesaron $846 \mathrm{~m}^{3}$ de residuos. La totalidad de los residuos fueron almacenados en el centro de El Cabril. No consta ningún transporte de residuos a instalaciones fuera de las fronteras del país (ENRESA, 2017). Las modalidades de transporte son variadas.

En Suecia, se procesan también más habitualmente residuos de media y baja intensidad. La modalidad de almacenamiento preferida es el almacenamiento en galerías subterráneas. Anualmente, se procesan 300 T de residuos, casi íntegramente ligadas a la producción energética, dado que los usos restantes son marginales. Dichos residuos se almacenan temporalmente en Simpevarp, en el entorno de la central de Oskarshamn, en una instalación denominada Clab (Instalación Central de Almacenamiento Temporal para Combustible Nuclear Utilizado) a la espera de que finalice la creación de un nuevo almacén de custodia definitiva en Forsmark, denominado SFR (Almacenamiento de Combustible Usado). La idea es mantener los residuos en el 
Clab hasta que el nuevo SFR esté operativo. El transporte de los residuos se realiza de forma marítima, por la localización costera de las centrales (Skala, 2008; SKB, 2016).

\subsection{Investigación, desarrollo e innovación}

Se ha determinado que la seguridad de las instalaciones nucleares depende de las innovaciones tecnológicas. Estas, a su vez, son fruto de la investigación, la innovación y el desarrollo (IEA, 2018). A nivel mundial se ha producido un descenso notable de la $\mathrm{I}+\mathrm{D}+\mathrm{i}$ nuclear.

En España, la mayor parte de la $\mathrm{I}+\mathrm{D}+\mathrm{i}$ nuclear está en manos del sector público y su presupuesto es desigual. Destacan los presupuestos que rondan el equivalente a 100 millones de EUR de 2017 a finales de la década de los años 1970 frente a los casi 10 millones en 2016. Es especialmente acusado el impacto de la crisis de 2009 sobre el presupuesto de $\mathrm{I}+\mathrm{D}+\mathrm{i}$, momento en el que tales actividades quedaron paralizadas.

En Suecia, la práctica totalidad de la I+D+i nuclear es pública. La evolución del presupuesto es más consistente y coherente: registra una disminución continuada desde la década de los años 1970. El presupuesto no acusa particularmente el impacto de la crisis de 2009. De hecho, los datos mantienen un descenso en sintonía con la tendencia decreciente de las últimas décadas.

\subsection{Opinión pública}

Es habitual que se elaboren barómetros de opinión ciudadana para estudiar las actitudes de la población respecto a la producción de energía nuclear, a la gestión de los residuos radiactivos y al marco institucional energético. La opinión pública es un indicador clave para determinar la evolución de la energía nuclear porque cabe esperar que las decisiones políticas sean coherentes con las opiniones ciudadanas.

Los usuarios españoles suelen mostrar una confianza moderada (40\%) acerca del marco regulatorio nuclear del país y de la actividad de los operadores nacionales (NEA, 2010). España es uno de los países de Europa en los que existe un mayor porcentaje de personas que creen que la energía nuclear no ayuda a luchar contra el cambio climático o que no lo saben con certeza (46\%) (Eurobarómetro, 2010). Sin embargo, respecto al último barómetro europeo, han aumentado en 11 puntos los partidarios de incrementar su importancia en el perfil energético. Asimismo, existe un empate (29\%) entre los partidarios de extender la vida de las centrales nucleares siempre que sean seguras y cumplan con las normativas internacionales y los partidarios de no practicar dicha prórroga. Los españoles prefieren que la localización de las nuevas centrales nucleares esté tan alejada del país como sea posible (36\%), antes que contar con una central española (23\%) o europea (21\%) para abastecer las necesida- 
des. España es el segundo país de la Unión en el que más se sobrestima el riesgo de un accidente nuclear (61\%). El 73\% de los encuestados creen que las centrales nucleares españolas son un riesgo para su bienestar. En estudios más recientes (Foratom, 2017), cerca del $60 \%$ de los españoles está en contra y aproximadamente el $30 \%$ a favor de la energía nuclear. El 38\% cree que los actuales reactores deberían clausurarse progresivamente y el $25 \%$, que deberían construirse nuevas instalaciones. Acerca de la gestión de residuos (Eurobarómetro, 2008), el 38\% de los españoles, un porcentaje superior a la media europea, se muestra reticente al almacenaje subterráneo. Desde el último barómetro, se ha evidenciado una pérdida de interés en las decisiones de localización de los almacenes. Más del 75\% de los españoles cree que dicho almacenaje debería regularse a nivel nacional. Sin embargo, más del $80 \%$ de los encuestados reconoce no haber recibido información sobre residuos nucleares.

En Suecia, las actitudes son notablemente diferentes. Los usuarios suecos tienen una confianza mayor en el marco regulatorio (NEA, 2010). El 75\% de los suecos confía en sus autoridades nacionales para gestionar los procesos nucleares (Eurobarómetro, 2010). El 73\% de los suecos considera que la fuente resulta positiva para luchar contra el cambio climático, el porcentaje más elevado de toda la Unión Europea. El 87\% considera que es útil para reducir la dependencia energética externa, el mayor consenso entre los países europeos. El 64\% cree que la energía nuclear ha servido para moderar y estabilizar la evolución de los precios, aunque el porcentaje de ciudadanos con esta creencia ha caído 7 puntos porcentuales desde el último barómetro. En lo referente al mantenimiento de las instalaciones, el $62 \%$ de los suecos respalda mantener en funcionamiento las centrales siempre que cumplan los estándares internacionales. De hecho, Suecia registra el segundo mayor respaldo (34\%) en la Unión para utilizar la nuclear como apoyo para desarrollar otras fuentes energéticas renovables. El 48\% de los suecos dejaría de apoyar inmediatamente la energía nuclear si no sirviera para complementar estas fuentes alternativas. La localización nacional de las instalaciones nucleares es importante para los ciudadanos: una mayoría (67\%) prefiere que las centrales se ubiquen en territorio nacional bajo supervisión de las autoridades suecas. Ligeramente más de la mitad opina que las ventajas de la fuente estudiada superan los costes a afrontar. El 65\% no percibe ningún riesgo para su bienestar en el funcionamiento de las centrales, proporción que ha aumentado en 7 puntos desde el último estudio. Los usuarios suecos suelen considerarse los mejor informados acerca de la fuente energética y suelen tener porcentajes elevados de acierto en preguntas sobre cultura nuclear. Sin embargo, la mitad reclama mayor información sobre la gestión de los residuos, aunque ya se consideran los europeos mejor informados al respecto. También se constata en Suecia una participación notable de organizaciones políticamente independientes y del Parlamento en el debate nuclear (Eurobarómetro, 2010). 
El apoyo mayoritario a la nuclear suele ser una tendencia consistente entre encuestas. En barómetros más recientes, el 68\% apoya mantener la energía nuclear. El 36\% es favorable a la construcción de nuevas centrales, especialmente si es para sustituir las infraestructuras envejecidas (el respaldo asciende al 44\%) y el 32\% cree que se deben mantener las instalaciones actuales hasta el final de su vida útil. Aproximadamente el $20 \%$ se opone a su utilización (Foratom, 2017). Finalmente, aproximadamente el $90 \%$ de los suecos creen que la planificación del depósito de materiales radiactivos es una medida de urgencia y prioridad. El $80 \%$ cree que no hay un nivel de seguridad óptimo alcanzable en el almacenamiento de residuos. Suecia es uno de los países de la Unión en los que el depósito de materiales en subsuelo recibe mayor apoyo (63\%). Las mayores preocupaciones de los suecos son los daños que los residuos pueden ocasionar sobre el medioambiente y la salud (41\%) y, de forma inusitada a nivel europeo, el transporte de los residuos hasta el lugar de almacenamiento (25\%). Los suecos son los más partidarios de contar con la supervisión de la Unión Europea y con la aprobación de los agentes locales para gestionar los residuos (Eurobarómetro, 2008).

\section{Conclusiones}

La energía nuclear es una fuente útil, pero problemática. Las ventajas que suelen atribuírsele son: la seguridad (de abastecimiento y operativa), la eficiencia, la competitividad vía precio, la flexibilidad y la sostenibilidad medioambiental. Mediante el análisis realizado, se ha comprobado que hay que proceder con cautela frente a estas ventajas, porque cada una de ellas lleva aparejada una inconveniencia que emerge ante un estudio más profundo de la realidad energética. Los inconvenientes encontrados son: el riesgo geopolítico asociado a la obtención de los combustibles nucleares, el elevado precio comparativo frente a tecnologías renovables, la posibilidad de sufrir accidentes con elevados costes materiales y personales, el envejecimiento de las instalaciones nucleares, la falsación de su aparente sostenibilidad cuando se observa el impacto intergeneracional de la operativa nuclear y los problemas que presentan la explotación y el procesamiento de combustibles nucleares, así como la oposición pública. Cada sociedad, en base a su situación y a sus preferencias, debe dar una respuesta ante el dilema configurado por estas ventajas e inconvenientes. España y Suecia son dos sociedades que han respondido al dilema. Entre ellas se aprecian semejanzas y diferencias.

Respecto a la introducción de la fuente nuclear en el perfil energético, España comenzó sus experimentos más tarde que Suecia y requirió mayor ayuda exterior, por su situación de precariedad fruto de la guerra y la dictadura. Suecia, por su parte, validó su posición ante el dilema nuclear mediante un referéndum, algo imposible en España por no encontrarse en un sistema democrático. En ambos casos, la razón 
para recurrir a la fuente nuclear fue el encarecimiento de otra fuente energética: el petróleo en España y la hidráulica en Suecia. Tanto España como Suecia intentaron autoabastecerse de combustible nuclear, pero no lo lograron y tuvieron que recurrir a importaciones con riesgo geopolítico asociado. Dicho riesgo es mayor para España que para Suecia, por la tipología de sus proveedores.

Una vez introducida la fuente, la elevada prevalencia nuclear en Suecia ha sido posible a causa de un cierto consenso político muy influido por la industria: incluso sus detractores suelen opinar que un abandono de la fuente debe producirse lentamente en el tiempo y siempre que se potencien las renovables. Indudablemente, los grupos de presión industriales favorables a la nuclear han tenido un profundo efecto tanto en España como en Suecia. En la actualidad, España y Suecia producen cantidades similares de energía nuclear, lo que, en relación con sus características socioeconómicas, le otorga un peso internacional y regional elevado a la nuclear sueca. Sin embargo, la producción sueca observada a lo largo del tiempo ha sido continuadamente decreciente hasta situarse en mínimos históricos, mientras que en España se ha mantenido más constante, en posición intermedia.

En términos geográficos, los criterios para el establecimiento de las instalaciones nucleares han sido distintos: España localiza sus centrales en áreas con menor riesgo sísmico y con proximidad a los ríos, mientras que Suecia usa el criterio de proximidad a la demanda y busca las zonas costeras. La producción entre centrales también difiere: España reparte la producción de forma igualitaria entre reactores, mientras que Suecia tiende a producir más en las centrales más envejecidas.

Respecto a las instalaciones, el envejecimiento de las mismas es un problema común, aunque parece preocupar menos en Suecia, cuyas centrales son más antiguas que las españolas y cuyo planteamiento es reconocer una vida útil de 60 años para las centrales más recientes, sobrepasando notablemente los tiempos planificados en España. La seguridad de estas instalaciones en su vida útil depende de la $\mathrm{I}+\mathrm{D}+\mathrm{i}$, en ambos países de eminente carácter público, aunque más constante y consistente en el caso de Suecia. La propiedad de las instalaciones presenta igualmente un componente diferencial: mientras en España existen varias empresas titulares de las instalaciones, en Suecia la titularidad la concentra principalmente una única empresa.

Desde un punto de vista técnico, el método de producción es más variado en Suecia que en España. En España esta producción complementa las fuentes fósiles y sólidas, mientras que en Suecia complementa a las renovables, paliando sus potenciales problemas sobre la seguridad de abastecimiento. Los residuos nucleares se almacenan también de manera distinta: en España se practica el almacenaje en superficie mientras que en Suecia se emplean técnicas subterráneas. 
Finalmente, la percepción social sobre la fuente estudiada es ampliamente más negativa en España que en Suecia, lo que determina la aparición de desutilidades en el consumo.

La fuente nuclear, al igual que las restantes fuentes energéticas, seguirá planteando posibilidades y retos en el futuro, lo que irá transformando las posibilidades de acción sobre el paradigma energético, así como los términos del dilema nuclear en España, en Suecia y a nivel global. Las próximas investigaciones al respecto deben centrarse en el estudio de las problemáticas sociales, económicas y ecológicas de los combustibles nucleares, y en el análisis de la vertiente operativa y sociológica de la fuente nuclear.

En clave institucional, se propone aumentar la transparencia de la fuente nuclear, incrementando la disponibilidad pública de información actualizada acerca de su operativa. Este trabajo ha tenido que lidiar con la opacidad que todavía predomina cuando se tratan cuestiones sensibles como la provisión del uranio o las emisiones contaminantes.

\section{Bibliografía}

ALLEN, T., BUSBY, J., MEYER, M., PETTI, D. (2010) Materials challenges for nuclear systems. Materials Today, 13, 14-23.

APERGIS, N., PAYNE, J. E. (2010) A panel study of nuclear energy consumption and economic growth. Energy Economics, 32, 545-549.

BALDWIN, D. A. (1997) The concept of security. Review of International Studies, 23(1), 5-26. Cambridge University Press.

BALTA-OZKAN, N., LE GALLO, J. (2018) Spatial variation in energy attitudes and perceptions: Evidence from Europe. Renewable and Sustainable Energy Reviews, $81,2160-2180$.

CARVAlHO, F. P., MADRUGA, M. J., REIS, M. C., AlVES, J. G., OliVEIRA, J. M., GOUVEIA, J., SILVA, L. (2007) Radioactivity in the environment around past radium and uranium mining sites of Portugal. Journal of Environmental Radioactivity, 96(1-3), 39-46.

CHERP, A., JEWELL, J. (2014) The concept of energy security: Beyond the four As. Energy Policy, 75, 415-421.

DAVIS, L., HAUSMAN, C. (2016) Market impacts of a nuclear power plant closure. American Economic Journal: Applied Economics, 8(2), 92-122.

ESPEJO MARÍN, C. (2002) La producción de electricidad de origen nuclear en España. Boletín de La Asociación de Geógrafos Españoles, (33), 65-77. 
FAWCETT, A. A., IYER, G. C., CLARKE, L. E., EDMONDS, J. A., HULTMAN, N. E., MCJEON, H. C., SHI, W. (2015) Can Paris pledges avert severe climate change? Science, 350(6265), 1168-1169.

FJAESTAD, M. (2013) The geopolitics of uranium : Swedish energy dependencies from a transnational perspective. En 24th International Congress of History of Science, Technology and Medicine. Manchester: Kungliga Tekniska Högskolan.

GRANDIN, K., JAGERS, P., KULLANDER, S. (2010) Nuclear Energy. AMBIO, 39(1), 26-30.

HEJAZI, R. (2017) Nuclear energy: Sense or nonsense for environmental challenges. International Journal of Sustainable Built Environment, 6(2), 693-700.

HÖGBERG, L. (2013) Root Causes and Impacts of Severe Accidents at Large Nuclear Power Plants. AMBIO, 42(3), 267-284.

HOLMBERG, S., HEDBERG, P. (2011) Party Influence on Nuclear Power Opinion in Sweden. Swedish National Election Studies Program, (5). Göteborgs Universitet.

HUHTALA, A., REMES, P. (2017) Quantifying the social costs of nuclear energy: Perceived risk of accident at nuclear power plants. Energy Policy, 105, 320-331.

JENKINS, J. D., ZHOU, Z., PONCIROLI, R., VILIM, R. B., GANDA, F., DE SISTERNES, F., BOTTERUD, A. (2018) The benefits of nuclear flexibility in power system operations with renewable energy. Applied Energy, 222, 872-884.

KABERGER, T. (2007) History of nuclear power in Sweden. Estudos Avançados, 21(59), 225-242.

KOSAI, S., UNESAKI, H. (2017) Quantitative analysis on the impact of nuclear energy supply disruption on electricity supply security. Applied Energy, 208, 1198-1207.

LARSSON, C. (1985) Historien om en svensk atombomb. Ny Teknik, (17-20).

LEE, C., CHIU, Y. (2011) Oil prices, nuclear energy consumption, and economic growth: New evidence using a heterogeneous panel analysis. Energy Policy, 39, 2111-2120.

LEIJONHUFVUD, S. (1994) Parentes?: en historia om svensk kärnkraft. Västerås: ABB Atom.

LENZEN, M. (2008) Life cycle energy and greenhouse gas emissions of nuclear energy: A review. Energy Conversion and Management, 49(8), 2178-2199.

LYMAN, E. S. (2011) Nuclear Energy and Human Health. In Encyclopedia of Environmental Health (pp. 185-192). Elsevier.

MARTÍNEZ-ALEGRÍA, R., SANZ, G., OLIVEIRA, S., MONTEQUI, I., CAMPOS, F. (2014) Spanish Nuclear Industry - Future perspectives and reserves' analysis. International Workshop "Uranium, Environment and Public Health," 8, 81-85.

MIR, M. C. (1999) Evaluación de los planes energéticos nacionales en España. Revista de Historia Industrial, (15), 161-178. 
MUDD, G. M., DIESENDORF, M. (2008) Sustainability of Uranium Mining and Milling: Toward Quantifying Resources and Eco-Efficiency. Environmental Science \& Technology, 42(7), 2624-2630.

PEARCE, J. M. (2012) Limitations of nuclear power as a sustainable energy source. Sustainability, 4(6), 1173-1187.

QVIST, S. A., BROOK, B. W. (2015) Environmental and health impacts of a policy to phase out nuclear power in Sweden. Energy Policy, 84, 1-10.

RUBIO-VARAS, M. M., DE LA TORRE, J. (2017) The Economic History of Nuclear Energy in Spain: Governance, Business and Finance. Cham: Springer International Publishing.

SAN MARTÍN, E., MUÑOZ, B., LORCA, A. (2014) Vulnerabilidad energética. En J. M. Blanco \& L. de la Corte (Eds.), Seguridad Nacional, amenazas y respuestas. Madrid: LID Editorial.

SANTOS-FRANCÉS, F., GIL PACHECO, E., MARTÍNEZ-GRAÑA, A., ALONSO ROJO, P., ÁVILA ZARZA, C., GARCÍA SÁNCHEZ, A. (2018) Concentration of uranium in the soils of the west of Spain. Environmental Pollution, 236, 1-11.

SKALA, B. (2008) Sweden's nuclear fuel cycle policy. In Technical Meeting on "Country Nuclear Fuel Cycle Profiles." Fukui.

STEINHAUSER, G., BRANDL, A., JOHNSON, T. E. (2014) Comparison of the Chernobyl and Fukushima nuclear accidents: A review of the environmental impacts. Science of The Total Environment, 470-471, 800-817.

SUMAN, S. (2018) Hybrid nuclear-renewable energy systems: A review. Journal of Cleaner Production, 181, 166-177.

TENGSTRÖM, E. (1990) Energin, makten och framtiden: samhällsvetenskapliga perspektiv på teknisk förändring. Estocolmo: Allmänna Förl.

TERÄVÄINEN, T., LEHTONEN, M., MARTISKAINEN, M. (2011) Climate change, energy security, and risk-debating nuclear new build in Finland, France and the UK. Energy Policy, 39, 3434-3442.

WITTNEBEN, B. (2012) The impact of the Fukushima nuclear accident on European energy policy. Environmental Science \& Policy, 15(1), 1-3.

ZOELLNER, T. (2009) Uranium: war, energy, and the rock that shaped the world. Londres: Viking, Penguin.

Informes, estadísticas y otros documentos institucionales

AGENCIA DE SUMINISTRO EURATOM (2016) Annual Report 2016. http:// ec.europa.eu/euratom/ar/last.pdf

ALFSEN, K., ESKELAND, G. (2007) A broader palette: The role of technology in climate policy. Estocolmo. Regeringskansliet.

APERC (2007) A Quest for Energy Security in the 21st Century: Resources and Constraints (No. \# 207-RE-01.2). Tokyo. http://aperc.ieej.or.jp/file/2010/9/26/

AURA ENERGY (2012) Updated scoping study further supports Häggån project viability. Melbourne. http://www.auraenergy.com.au/assets/ 
BERTEL, E. (2005) Nuclear energy and the security of energy supply. NEA News, 23(2), 4-7. https://www.oecd-nea.org/pub/newsletter/2005/23-2-securitysupply.pdf

CONSEJO DE LA UE. Directivas 2011/70/Euratom, 2009/71/Euratom y 2013/59/ Euratom. Diario Oficial de la Unión Europea.

CSN (2015) La energía nuclear. Madrid. https://www.csn.es/documents/

de las Heras Abás, A. (2016) Informe de situación de las emisiones de CO2 en el mundo. Barcelona. Fundación Empresa y Clima.

ENRESA (2017). Informe Anual. Madrid. http://www.enresa.es/documentos/ informe_anual_2016.pdf

EURATOM (2012). Diario Oficial de la Unión Europea.

EUROBARÓMETRO (2008). Attitudes towards radioactive waste. (2010) Europeans and Nuclear Safety. http://ec.europa.eu/commfrontoffice/publicopinion/ archives/ebs/

EUROSTAT (2018). Energy Database. http://ec.europa.eu/eurostat/web/energy/data FORATOM (2017). What People Really Think about Nuclear Energy. Atw International Journal for Energy Power, 62(3). https://www.kernenergie.de/

FORO DE LA INDUSTRIA NUCLEAR ESPAÑOLA (2016). Comentario de informes. https://www.foronuclear.org/es/

IEA (2018) World energy statistics. París. https://www.iea.org/statistics

INSTITUTE FOR ECONOMICS AND PEACE (2017). Global Peace Index. Sydney. http://visionofhumanity.org/app/uploads/2017/06/GPI17-Report.pdf

MINISTERIO DE ENERGÍA, TURISMO Y AGENDA DIGITAL DE ESPAÑA (2018) Energía nuclear. http://www.minetad.gob.es/energia/nuclear/

MUÑOZ MANSILLA, M. (2015) Contribución de la Energía Nuclear al Cambio Climático. Madrid.https://www.sne.es/images/stories/recursos/actividades/

NEA, IEA, OECD (2015) Technology Roadmap: Nuclear Energy. París. https://www. oecd-nea.org/pub/techroadmap/techroadmap-2015.pdf

NEA (2010) Public Attitudes to Nuclear Power. https://www.oecd-nea.org/ndd/pubs/

Observatorio Nuclear Europeo. (2018). Prices. http://ec.europa.eu/euratom/ observatory_price.html

SEGOVIANO MONTERRUBIO, S. (2011) España ante el reto de la seguridad energética (No. 56/2011). Fundación Alternativas.

SKB (2016) The Swedish System. http://www.skb.com/our-operations

SNE (2018) Origen del uranio. https://www.sne.es/es/

TEULE, R. (2011) ¿Por qué Greenpeace le dice NO a la energía nuclear? Greenpeace Internacional. http://www.greenpeace.org/

WNA (2018) Country profiles. http://www.world-nuclear.org/

WWF (2009) Climate Scorecard Sweden. http://www.wwf.se/ 